\title{
ROJ
}

\section{Initial clinical outcomes of proton beam radiotherapy for hepatocellular carcinoma}

Jeong II Yu, MD, PhD1', Gyu Sang Yoo, MD1', Sungkoo Cho, PhD1', Sang Hoon Jung, PhD1, Youngyih Han, PhD',2, Seyjoon Park, MS 1 , Boram Lee, PhD ${ }^{1}$, Wonseok Kang, MD, PhD ${ }^{3}$, Dong Hyun Sinn, MD, PhD ${ }^{3}$, Yong-Han Paik, MD, PhD ${ }^{3}$, Geum-Youn Gwak, MD, PhD ${ }^{3}$, Moon Seok Choi, MD, PhD ${ }^{3}$, Joon Hyeok Lee, MD, PhD³, Kwang Cheol Koh, MD, PhD ${ }^{3}$, Seung Woon Paik, MD, PhD ${ }^{3}$, Hee Chul Park, MD, PhD ${ }^{1,2}$

'Department of Radiation Oncology, Samsung Medical Center, Sungkyunkwan University School of Medicine, Seoul; ${ }^{2}$ Department of Medical Device Management and Research, Samsung Advanced Institute for Health Sciences and Technology

(SAIHST), Sungkyunkwan University, Seoul;

${ }^{3}$ Department of Medicine, Samsung Medical Center, Sungkyunkwan University School of Medicine, Seoul, Korea

Purpose: This study aimed to evaluate the initial outcomes of proton beam therapy (PBT) for hepatocellular carcinoma (HCC) in terms of tumor response and safety.

Materials and Methods: HCC patients who were not indicated for standard curative local modalities and who were treated with PBT at Samsung Medical Center from January 2016 to February 2017 were enrolled. Toxicity was scored using the Common Terminology Criteria for Adverse Events (CTCAE) version 4.0. Tumor response was evaluated using modified Response Evaluation Criteria in Solid Tumors (mRECIST).

Results: A total of 101 HCC patients treated with PBT were included. Patients were treated with an equivalent dose of 62-92 GyE $E_{10}$. Liver function status was not significantly affected after PBT. Greater than $80 \%$ of patients had Child-Pugh class A and albuminbilirubin (ALBI) grade 1 up to 3-months after PBT. Of 78 patients followed for three months after PBT, infield complete and partial responses were achieved in 54 (69.2\%) and 14 (17.9\%) patients, respectively.

Conclusion: PBT treatment of HCC patients showed a favorable infield complete response rate of $69.2 \%$ with acceptable acute toxicity. An additional follow-up study of these patients will be conducted.

Keywords: Hepatocellular carcinoma, Proton, Radiotherapy, Response, Toxicity

\section{Introduction}

Hepatocellular carcinoma (HCC) is the most common liver cancer in adults and one of the most common causes of cancer-related death worldwide [1]. Recently, improvements in HCC treatment and overall survival have been made $[2,3]$.
However, many HCC patients are still diagnosed at an advanced stage of the disease not suitable for curative local modalities such as surgery or radiofrequency ablation (RFA). Transarterial chemoembolization (TACE) is commonly performed in such cases and improves survival, but is unsatisfactory in terms of local control rate $[4,5]$. Advanced radiotherapy (RT) treatment

Received 16 August 2017, Revised 17 October 2017, Accepted 15 November 2017.

Correspondence: Hee Chul Park, MD, PhD, Department of Radiation Oncology, Samsung Medical Center, Sungkyunkwan University School of Medicine, 81 Irwon-ro, Gangnam-gu, Seoul 06351, Korea. Tel: +82-2-3410-2612, Fax: +82-2-3410-2619, E-mail: hee.ro.park@skku.edu

(c) This is an Open Access article distributed under the terms of the Creative Commons Attribution Non-Commercial License (http://creativecommons.org/ licenses/by-nc/4.0/) which permits unrestricted non-commercial use, distribution, and reproduction in any medium, provided the original work is properly cited.

www.e-roj.org 
techniques such as intensity-modulated RT and stereotactic body ablative RT (SABR) or stereotactic body RT with imageguidance and/or respiration gating have also been applied to HCC management [6]. Although favorable outcomes have been reported, toxicity to the liver and gastroduodenum remains a problem [7-9]. Especially, there is still concern about the dreadful toxicity of radiation-induced liver disease (RILD) [10]. Proton beam RT (PBT) has recently been expanded to treat cancer with high conformity and precision by exploiting its unique dose-depth profile, which permits a sharp increase in dose at a certain depth, known as a "Bragg-peak" [11]. PBT could allow effective treatment of HCC while preserving surrounding normal liver tissue [12].

Samsung Medical Center started applying PBT for cancer management in 2016, with highest priority indications given to pediatric cancer and adult HCC patients [13]. Here we present initial results in terms of tumor response and safety for 101 HCC patients treated with PBT at Samsung Medical Center for 14 months.

\section{Materials and Methods}

\section{Proton therapy center and patients}

The PBT center at Samsung Medical Center has been described previously [13]. All patients who received PBT were registered and managed as a prospective cohort approved by the Samsung Medical Center Institutional Review Board (No. 201510-135). Subjects had unresectable HCC diagnosed according to the American Association for the Study of Liver Diseases guidelines [14] and were treated with PBT in the study period, from January 2016 to February 2017.

Proton therapy was preferentially considered in the following cases that were not eligible for surgery and RFA, and was finally determined through multidisciplinary discussion. First, patients who consented to participate in our proton therapy clinical trials and who registered at clinicaltrials.gov (NCT02632864 and NCT02571946) were considered. Second, for patients indicated for SABR in our institution, treatment was determined considering the margin (range uncertainty and setup uncertainty) of proton beam and liver volume exposures greater than $24 \mathrm{~Gy}$ according to the results of previous studies $[15,16]$. Patients with lesions exceeding $3 \mathrm{~cm}$, in which $S A B R$ was not indicated, but who had the potential for cure with one or two lesions were considered for PBT. Lastly, all patients indicated for PBT were reaffirmed for PBT appropriateness through respiratory training. If the optimal respiratory control was not possible, general anesthesia and usage of a ventilator
Table 1. Baseline characteristics of 101 patients

\begin{tabular}{|c|c|}
\hline Characteristic & Value \\
\hline Age (yr) & $63(35-91)$ \\
\hline \multicolumn{2}{|l|}{ Sex } \\
\hline Male & $87(86.1)$ \\
\hline Female & $14(13.9)$ \\
\hline \multicolumn{2}{|l|}{ ECOG performance status } \\
\hline 0 & $52(51.5)$ \\
\hline 1 & $44(43.6)$ \\
\hline 2 & $5(5.0)$ \\
\hline \multicolumn{2}{|l|}{ Etiology } \\
\hline HBV & $81(80.2)$ \\
\hline $\mathrm{HCV}$ & $8(7.9)$ \\
\hline Alcohol & $3(3.0)$ \\
\hline Other & $9(8.9)$ \\
\hline \multicolumn{2}{|l|}{ Liver cirrhosis } \\
\hline Yes & $74(73.3)$ \\
\hline No & $27(26.7)$ \\
\hline \multicolumn{2}{|l|}{ Child-Pugh class } \\
\hline A5 & $73(72.3)$ \\
\hline A6 & $17(16.8)$ \\
\hline B7 & $5(5.0)$ \\
\hline B8 & $3(3.0)$ \\
\hline B9 & $2(2.0)$ \\
\hline C10 & $1(1.0)$ \\
\hline \multicolumn{2}{|l|}{ ALBI grade } \\
\hline 1 & $89(88.1)$ \\
\hline 2 & $11(10.9)$ \\
\hline 3 & $1(1.0)$ \\
\hline AFP (ng/mL) & $17.4(1.3-200,000)$ \\
\hline Maximum tumor diameter $(\mathrm{cm})$ & $2.5(1.0-16.0)$ \\
\hline \multicolumn{2}{|l|}{ Tumor multiplicity } \\
\hline No & $74(73.3)$ \\
\hline Yes & $27(26.7)$ \\
\hline \multicolumn{2}{|l|}{ Portal vein tumor thrombosis } \\
\hline No & $72(71.3)$ \\
\hline Yes & $29(28.7)$ \\
\hline \multicolumn{2}{|l|}{ Modified UICC T stage } \\
\hline $\mathrm{T} 1$ & $36(35.6)$ \\
\hline $\mathrm{T} 2$ & $24(23.8)$ \\
\hline T3 & $31(30.7)$ \\
\hline T4 & $10(9.9)$ \\
\hline \multicolumn{2}{|l|}{ Previous treatment (repeated measure) } \\
\hline Surgical resection & $18(17.8)$ \\
\hline Transplantation & $1(1.0)$ \\
\hline Radiofrequency ablation & $39(38.6)$ \\
\hline Trans-arterial chemoembolization & $97(96.0)$ \\
\hline Radiotherapy & $16(15.8)$ \\
\hline Sorafenib & $3(3.0)$ \\
\hline None & $4(4.0)$ \\
\hline \multicolumn{2}{|l|}{ Respiratory motion control } \\
\hline Breath hold & $74(73.3)$ \\
\hline Respiratory-gated & $10(9.9)$ \\
\hline
\end{tabular}

to be next page 
Table 1. Continued

\begin{tabular}{lc}
\hline \multicolumn{1}{c}{ Characteristic } & Value \\
\hline Motion encompassing & $16(15.8)$ \\
Mechanical ventilation & $1(1.0)$ \\
Dose scheme (EOD2) & \\
5 CGE/10 fx (62 CGE) & $25(24.8)$ \\
6 CGE/10 fx (80 CGE) & $2(2.0)$ \\
6.6 CGE/10 fx (92 CGE) & $61(60.4)$ \\
48 CGE/6 fx (72 CGE) & $3(3.0)$ \\
Others ${ }^{\text {a) }}$ & $10(9.9)$ \\
Purpose of treatment & \\
Consolidative & $31(30.7)$ \\
Salvage & $49(48.5)$ \\
Definitive & $3(3.0)$ \\
Palliative & $18(17.8)$ \\
\hline
\end{tabular}

Values are presented as median (range) or number (\%). ECOG, Eastern Cooperative Oncology Group; ALBI, albumin-bilirubin; AFP, alpha-fetoprotein; UICC, Union for International Cancer Control; EQD2, equivalent dose in $2 \mathrm{~Gy}$; CGE, cobalt gray equivalent.

a) The detailed dose scheme is as follows: 12 CGE $\times 4$ (1), 12 CGE $\times$ 5 (2), $10 \mathrm{CGE} \times 5$ (1), 5 CGE $\times 15$ (1), 4 CGE $\times 10$ (2), $3.3 \mathrm{CGE} \times 22$ (1), $3 \mathrm{CGE} \times 11(1)$, and $3 \mathrm{CGE} \times 10$ (1).

was recommended.

For patients participating in prospective clinical trials, if the tumor was far from the liver dome and did not have proper fiducial marker (e.g., densely iodized oil or surgical clip), gold marker insertion was considered before PBT simulation.

\section{Respiration motion management}

If PBT was considered an appropriate modality by a radiation oncologist, audiovisual-guided respiration training was performed prior to computed tomography (CT) scanning. The breathing performance of each patient was monitored, and the patient was trained using an in-house biofeedback system that correlates breathing with a time-amplitude curve seen by the patient using liquid crystal display (LCD) glasses. Two breathing techniques, breath-holding and voluntary shallow breathing, are currently used for liver treatment, and the proper technique for each patient was chosen either by a physician for clinical reasons before respiration training or by a respiration trainer after checking the patient's respiration performance. In the breath-holding technique, an individually achievable breath-holding guideline is set, and a visual prompt on the display informs the patient when to hold the breath. In the shallow breathing technique, the patient is guided by an upper inhalation line and an exhalation baseline set after monitoring voluntary shallow breathing for several minutes. After choosing a breathing technique, the patient's respiration was monitored using the in-house biofeedback system and an Anzai amplitude based respiration monitoring system (AZ-733; Anzai Medical Co. Ltd., Tokyo, Japan) in the CT scanning and PBT treatment room.

Because PBT has the problem of a large range uncertainty depending on the heterogeneity of each tissue, the breathhold technique was mandatory and respiratory-gated or mechanical ventilator application is considered as the next option. PBT using a motion encompassing method is finally considered in highly limited cases of poor respiratory control with centrally located tumors and a minor range uncertainty.

\section{Proton therapy planning and delivery}

In patients treated with the breath-holding technique, six CT scans were performed: twice before injecting contrast agent, twice (arterial and portal) after injecting contrast agent, and twice to check for inter-fraction motion variation caused by patient breathing. Generally the second CT image before injecting the contrast agent was used for PBT treatment planning.

In patients treated with respiratory-gated and/or mechanical ventilation or the motion encompassing method, four-dimensional CT images were acquired using the biofeedback system described above to regulate breathing. The maximum intensity projection image for planning the motion encompassing method, and end-exhale phase image for respiratory-gated and/or mechanical ventilation were used as the primary images for PBT treatment planning.

Gross tumor volume (GTV) was outlined on non-enhanced $\mathrm{CT}$ images using reference contrast-enhanced $\mathrm{CT}$ and magnetic resonance (MR) images. An additional margin of $0.5 \mathrm{~cm}$ was added to the GTV to define the clinical target volume (CTV), and an additional margin of $0.5 \mathrm{~cm}$ was applied to the CTV to define the planning target volume (PTV). For the planning of motion encompassing method, internal target volume defined as all phase summation of CTV was used and an additional $0.5 \mathrm{~cm}$ was added for PTV. The treatment plan using multibeam PBT was made with the goal of delivering 66 cobalt gray equivalents (CGE; multiply proton physical dose by relative biological effectiveness of 1.1) in 10 fractions over a period of 2 weeks. However, a total dose of 50 CGE was administered when PBT was performed for consolidation after TACE. Liver treatment plans were created using a treatment planning system (RaySearch Laboratories, Stockholm, Sweden), taking into account the uncertainty of the dose range and choosing 
beam directions to avoid normal liver as much as possible and to avoid organs-at-risk such as the stomach, bowels, and skin. For the wobbling beam method, parameters including proton range, compensator, modulation width of spread-out Bragg peak (SOBP), and block were modified and evaluated considering target coverage and motion. The line scanning beam which is performed only in breath-hold technique method worked with given constraints for the same planning goals. Finally, every plan evaluated the plan robustness on range uncertainty $( \pm 3.5 \%)$ and setup uncertainty $(x, y, z$ shift: $\pm 3 \mathrm{~mm}$ ) using the perturbed dose function in treatment planning system.

Before the first treatment, every plan performed the pretreatment quality assurance for verification of monitor unit (MU) and depth dose distribution on wobbling beam, and point dose and two-dimensional (2D) dose distribution on scanning beam. For all cases, the measured MU and point dose were less than 3\% different from the calculated values. Comparing with the calculated values of treatment planning system and measured values, the range of proton beam was less than 1 $\mathrm{mm}$ and the 2D dose distribution was more than 95\% passing rate for 2\%/2 mm gamma index.

Before each treatment session, daily image guidance was conducted using cone beam CT and/or orthogonal digital radiography acquired using VeriSuite (MedCom, Darmstadt, Germany). Tumor localization was evaluated by comparing the positions of fiducial markers including densely iodized oils, surgical clip, liver dome, and gold markers.

\section{Follow-up}

Weekly interviews and a last-week laboratory test were conducted during the PBT treatment period. The first follow-up evaluation was performed at one month after completion of PBT. Subsequent follow-up evaluations were performed every 2-3 months if disease progression was not detected. At each follow-up, PBT response was evaluated using the modified Response Evaluation Criteria in Solid Tumor (mRECIST) [17], and toxicity was scored using Common Terminology Criteria for Adverse Events (CTCAE) version 4.0. The objective response rate was calculated based on a combination of complete response (CR) and partial response (PR).

RILD which separated into 'classic', defined as alkaline phosphatase increases more than two times the normal level but level of transaminase, bilirubin and ammonia remain normal and 'non-classic', as elevated liver transaminases more than five times the upper normal limit or decline in liver function (measured by a worsening of Child-Pugh score by 2

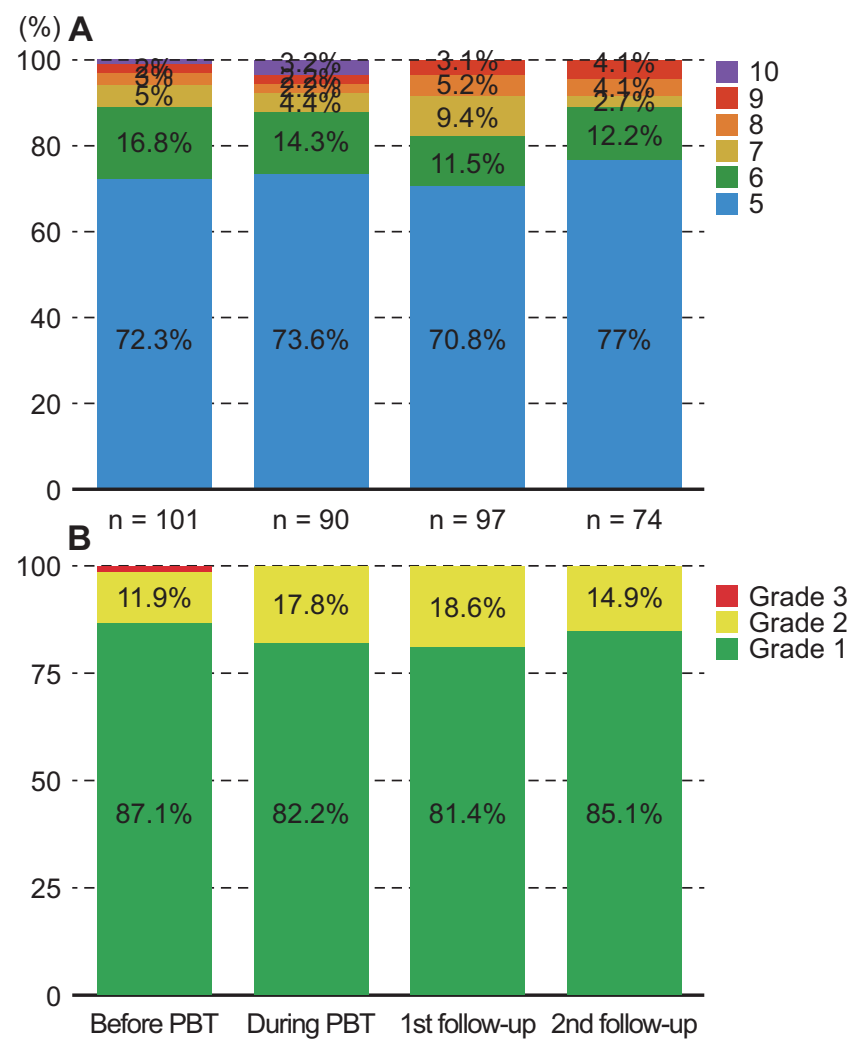

Fig. 1. Changes in liver function status. Liver function was assessed by Child-Pugh score (A) and the albumin-bilirubin grade (B) before proton beam therapy (PBT), during PBT, and at 1- and 3-month follow-up visits after completion of PBT.

or more) were also evaluated.

Esophagogastroduodenoscopy (EGD) was recommended to all enrolled patients for baseline evaluation before starting PBT except when the distance between the tumor and stomach or duodenum was larger than $5 \mathrm{~cm}$. Follow-up EGD was not routinely performed for all patients treated with PBT, except for patients with epigastric pain and/or bowel exposure to radiation.

\section{Results}

\section{Patients}

A total of 103 HCC patients were enrolled in the Samsung Cnn Proton Center Registry from January 2016 to February 2017. Two patients were excluded from the analysis. One patient received $\mathrm{PBT}$ for re-irradiation to the para-aortic lymph node and the other patient showed rapid intra- and extrahepatic metastasis before PBT treatment began.

The characteristics of the remaining 101 patients are 
Table 2. Baseline status and acute toxicity profile of PBT at 3-month follow-up after PBT

\begin{tabular}{|c|c|c|c|c|}
\hline & Grade I & Grade II & Grade III & Grade IV \\
\hline \multicolumn{5}{|c|}{ Baseline status before PBT } \\
\hline Anemia & $50(49.5)$ & $6(5.9)$ & - & - \\
\hline Leukopenia & 14 (13.9) & $5(5.0)$ & $3(3.0)$ & - \\
\hline Thrombocytopenia & $45(44.6)$ & $9(8.9)$ & $10(9.9)$ & - \\
\hline AST & $28(27.7)$ & $3(3.0)$ & - & - \\
\hline ALT & $17(16.8)$ & $4(4.0)$ & - & - \\
\hline ALP & $28(27.7)$ & - & - & - \\
\hline Hypoalbuminemia & $8(7.9)$ & $9(8.9)$ & - & - \\
\hline Hyperbilirubinemia & $5(5.0)$ & $9(8.9)$ & $2(2.0)$ & - \\
\hline Anorexia & $2(2.0)$ & - & - & - \\
\hline Nausea & $2(2.0)$ & - & - & - \\
\hline Vomiting & - & - & - & - \\
\hline Diarrhea & - & - & - & - \\
\hline Abdominal pain & $3(3.0)$ & - & - & - \\
\hline \multicolumn{5}{|c|}{ Acute toxicity after 3-month follow-up } \\
\hline Anemia & $57(56.4)$ & $3(3.0)$ & $2(2.0)$ & - \\
\hline Leukopenia & $25(24.8)$ & $20(19.8)$ & $3(3.0)$ & - \\
\hline Thrombocytopenia & $48(47.5)$ & $25(24.8)$ & $10(9.9)$ & - \\
\hline AST & 40 (39.6) & $2(2.0)$ & $1(1.0)$ & - \\
\hline ALT & $25(24.8)$ & $4(4.0)$ & $1(1.0)$ & - \\
\hline ALP & $35(34.7)$ & $2(2.0)$ & - & - \\
\hline Hypoalbuminemia & $16(15.8)$ & $9(8.9)$ & - & - \\
\hline Hyperbilirubinemia & $11(10.9)$ & $12(11.9)$ & $4(4.0)$ & $1(1.0)$ \\
\hline Anorexia & $12(11.9)$ & $1(1.0)$ & - & - \\
\hline Nausea & $3(4.3)$ & $2(2.0)$ & - & - \\
\hline Vomiting & $5(5.0)$ & - & - & - \\
\hline Diarrhea & - & - & - & - \\
\hline Abdominal pain & $10(9.9)$ & $3(3.0)$ & - & - \\
\hline Dermatitis & 19 (18.8) & $5(5.0)$ & - & - \\
\hline
\end{tabular}

Values are presented as number (\%).

PBT, proton beam therapy; AST, aspartate aminotransferase; ALT, alanine aminotransferase; ALP, alkaline phosphatase.

displayed in Table 1. Two patients were treated with PBT twice, one because of a synchronous multiple intrahepatic tumor, and another because of a metachronous outfield intrahepatic recurrence. The breath-holding technique for respiratory motion management was used in roughly three-quarters of the patients. Respiratory-gated or mechanical ventilation that treats the target during a specific respiratory cycle including end-exhale phase were used in 10 (9.9\%) patients and one (1.0\%) patient, respectively. The motion encompassing method that irradiates the target during all respiratory cycles was used in $16(15.8 \%)$ patients. Ten fractions of 6.6 CGE was the prescribed dose scheme for $>60$ patients. As an equivalent dose in 2 Gy (EQD2) calculated using an $\alpha / \beta$ ratio of 10 for acute response tissues, a dose of 62-92 CGE was used in 97 patients (96.0\%).

\section{Safety of PBT}

During the follow-up, classic RILD was not detected in the enrolled patients and non-classic RILD measured by a worsening of Child-Pugh score by 2 was developed in three patients (3.0\%) at one month and additional one patient (1.0\%) at three months after PBT completion. Among them, one was related to multiple intrahepatic progression, and three patients showed normalized results at the next follow-up.

Changes in liver function status were assessed by ChildPugh score and albumin-bilirubin (ALBI) grade before and during PBT and at 1- and 3-month follow-up visits after completion of PBT (Fig. 1). Child-Pugh score generally did not significantly change during PBT, was slightly elevated at 1-month follow-up visits, and tended to return almost to baseline at 3-month follow-up visits (Fig. 1A). The percentage of Child-Pugh class A was $90.1 \%$ before PBT, $87.9 \%$ during 
EGD befor PBT $(n=76)$

(repeated measure)

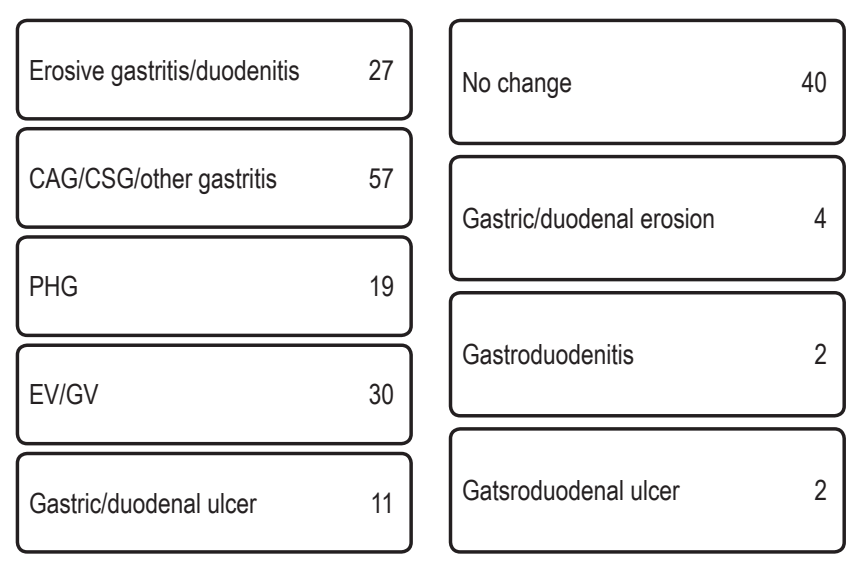

Fig. 2. Esophagogastroduodenoscopy (EGD) findings before and after proton beam therapy (PBT). Two newly developed gastroduodenal ulcers were detected by EGD during follow-up, and one patient showed melena and hematochezia 3 months after PBT. CAG, chronic atrophic gastritis; CSG, chronic superficial gastritis; PHG, portal hypertensive gastropathy; EV, esophageal varices; GV, gastric varices.

PBT, 82.3\% at 1-month and 89.2\% at 3-month follow-up after PBT completion. ALBI grade showed a similar pattern and the percentage of $\mathrm{ALBI}$ grade 1 was $87.1 \%$ before PBT, $82.2 \%$ during PBT, $81.4 \%$ at 1 -month and $85.1 \%$ at 3 -month followup after PBT completion (Fig. 1B).

Other acute toxicities possibly related to PBT treatment are listed in Table 2. Generally, there was no noticeable difference in treatment-related toxicity profiles compared with baseline. Grade III or higher toxicities were not detected except for one grade IV hyperbilirubinemia. This patient suffered hepatic failure associated with cirrhosis, showed repeated intrahepatic tumor recurrence, and underwent PBT as a bridging therapy prior to liver transplantation. The patient received a liver transplantation from a deceased donor on the 10th day after 10 fractions of PBT and has been on follow-up with no evidence of tumor recurrence.

Fig. 2 illustrates the EGD results before and after PBT. During the follow-up period after completion of PBT, two cases (2.0\%) of newly developed gastroduodenal ulcers were detected by EGD. In one case that had a more than $6 \mathrm{~cm}$ sized HCC in S4 and was treated with a total dose of 66 CGE in 10 fractions under the respiratory-gated technique, the gastric ulcer was found at 1 month after PBT completion within the irradiation site although the patient had no symptomatic complaints. The maximum stomach dose to the patient was 43.2 CGE in the PBT plan. In the other case, larger than $10 \mathrm{~cm}$ sized HCC in S4 was treated to a total dose of 60 CGE in 10 fractions using the breath-holding technique. Patient developed melena and hematochezia after PBT. Emergency EGD at approximately 100 days after PBT completion confirmed a duodenal ulcer located within the PBT site (Fig. 3). The maximum duodenal dose to the patient was 27.6 CGE in the PBT plan.

In six other cases, gastroduodenal changes including erosion and/or inflammation were found; in three of these cases, the changes were located within the PBT irradiation field. Therefore, the total number of patients with PBT-induced gastroduodenal toxicity was five (5.0\%).

\section{Tumor response following PBT}

Tumor response rates at 1 and 3 months after completion of PBT are displayed in Table 3. At the first follow-up evaluation for infield tumor response of 101 patients, CR was achieved in 45 patients (44.6\%), PR in 25 (24.8\%), and progressive disease (PD) in two (2.0\%). Among the two cases assessed as PD, one case showed a main lesion larger than $14 \mathrm{~cm}$ with multiple metastatic nodules and was treated with 10 fractions of 50 CGE to the dominant lesion. The other case had received 10 fractions of 66 CGE for $4.6 \mathrm{~cm}$ lesions with left main portal vein tumor thrombosis (PVTT). In this case, the main lesion was assessed as PR but PVTT was assessed as PD.

Table 3. Tumor response at 1- and 3-month follow-up after PBT

\begin{tabular}{|c|c|c|c|c|}
\hline & CR & PR & SD & PD \\
\hline \multicolumn{5}{|c|}{ Follow-up at 1 month $(n=101)$} \\
\hline Infield & 45 (44.6) & $26(25.7)$ & $28(27.7)$ & $2(2.0)$ \\
\hline Overall & $38(37.6)$ & $19(18.8)$ & $25(24.8)$ & 19 (18.8) \\
\hline \multicolumn{5}{|c|}{ Follow-up at 3 months $(n=78)$} \\
\hline Infield & $54(69.2)$ & $14(17.9)$ & $8(10.3)$ & $2(2.6)$ \\
\hline Overall & $42(53.8)$ & $8(10.3)$ & $4(5.1)$ & $24(30.8)$ \\
\hline
\end{tabular}

Values are presented as number (\%).

PBT, proton beam therapy; $C R$, complete response; PR, partial response; SD, stable disease; PD, progressive disease. 


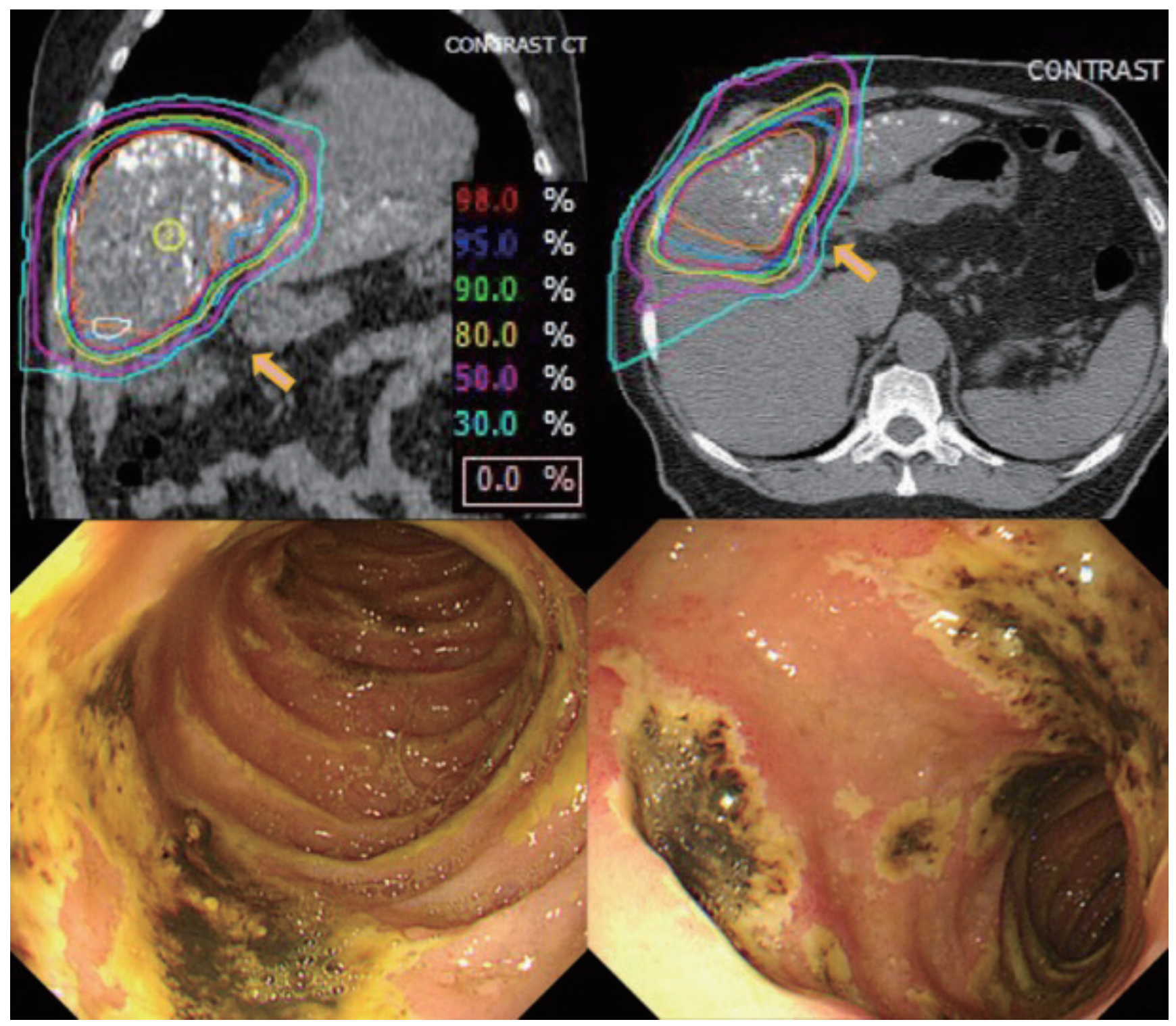

Fig. 3. Proton beam therapy (PBT)-related duodenal ulcer. Isodose curves of PBT planning and esophagogastroduodenoscopy detection of a duodenal ulcer.

Of the 78 patients followed up for three months after PBT for infield tumor response, CR was achieved in 54 patients (69.2\%). Overall CR and PR were obtained in 42 (53.8\%) and 8 (12.8\%) patients, respectively.

The median follow-up period for all enrolled patients was 4.9 months (range, 1.3 to 14.6 months). Accumulated CR rate as maximum infield response according to follow-up period is displayed in Fig. 4. Several cases showing CR after PBT are shown in Fig. 5.

During follow-up, local PD was detected in six cases (5.9\%), with a median time between PBT and local PD of 2.7 months (range, 1.3 to 7.3 months).

\section{Discussion and Conclusion}

In the present study, we evaluated the tumor response and safety of $101 \mathrm{HCC}$ patients treated with PBT for 14 months and observed favorable outcomes of infield CR in $69.2 \%$ of patients and overall CR in 53.8\%, with acceptable toxicity.

Despite a lack of supporting evidence based on randomized trials, advanced HCC is often treated using local modalities such as RFA, TACE, and surgical resection [18-20]. In HCC, local treatment of the primary lesion is important for preservation of surrounding normal liver function, which deteriorates with tumor progression and often with treatment of the tumor 

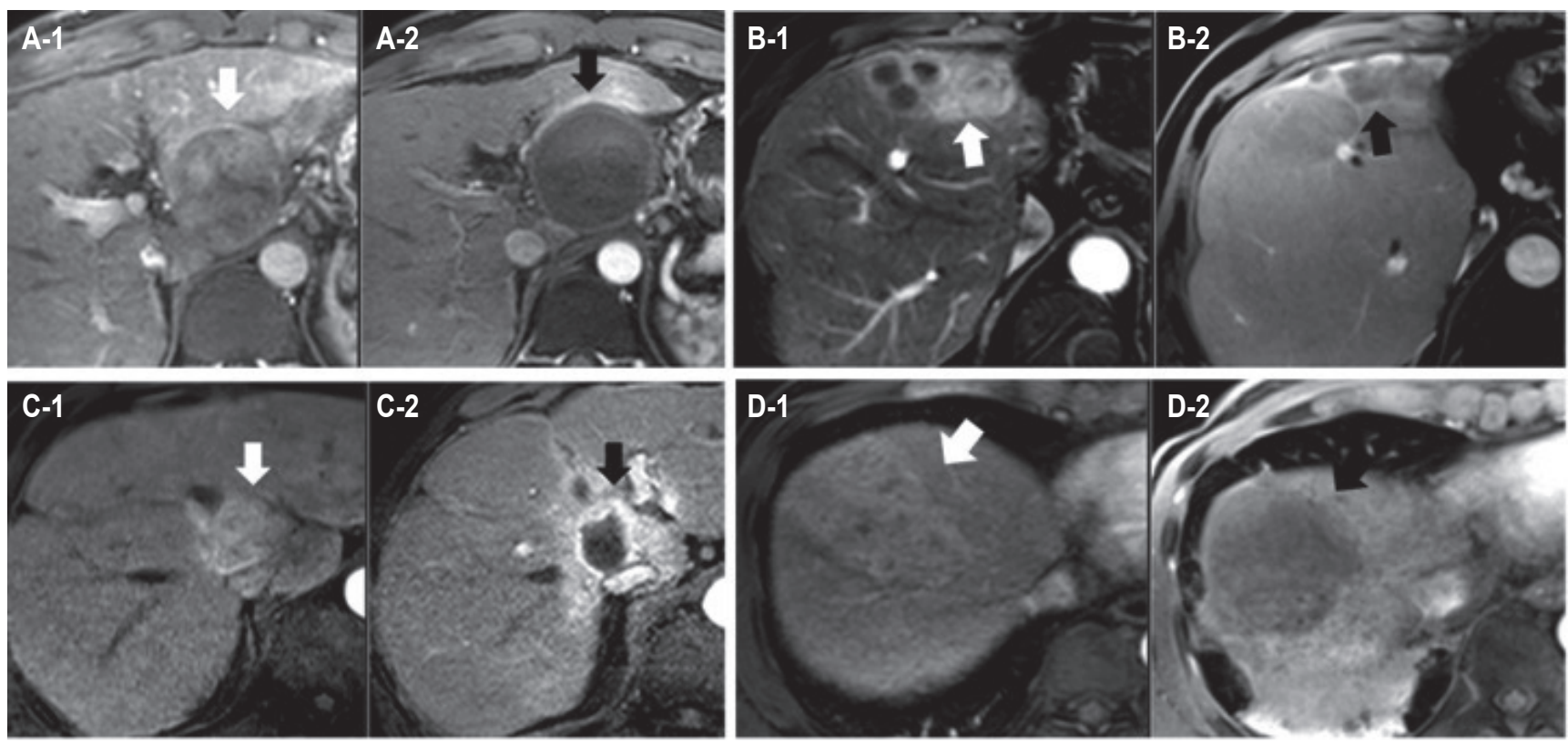

Fig. 4. Accumulated complete response rate as maximum infield response according to mRECIST criteria. The characteristic arterial phase enhancement of hepatocellular carcinoma (white arrows) was disappeared after proton beam therapy (black arrows).

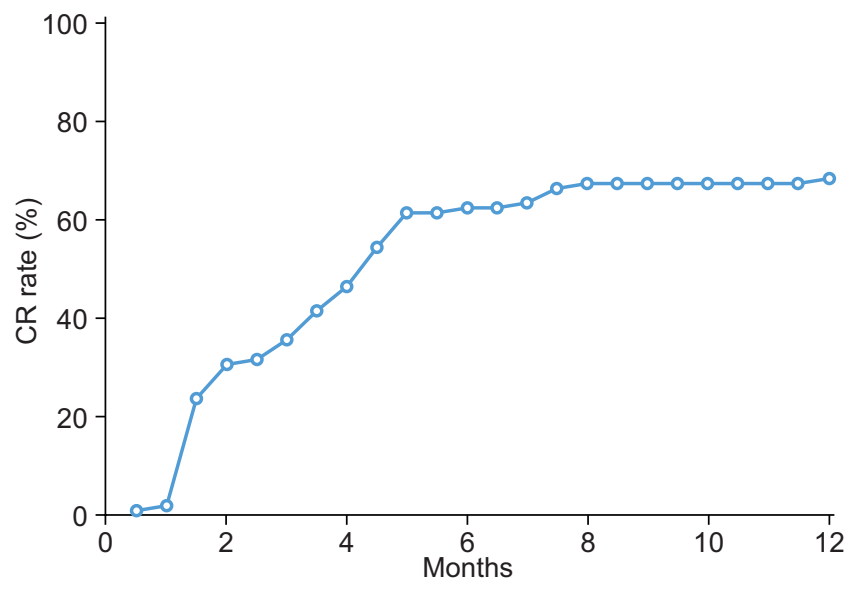

Fig. 5. Several cases showing complete response (CR) according to the modified Response Evaluation Criteria in Solid Tumors (mRECIST) criteria after proton beam therapy.

itself [21]. Indeed, these local treatments have proven useful in certain HCC patients [22-24].

RT is one of the most useful local modalities in oncology. Although the role of RT in HCC management has been limited historically, RT usage for HCC has increased recently with development of new RT techniques [25]. PBT, which uses a beam of charged particles to irradiate tissue, has a unique characteristic known as a 'Bragg peak', in which the maximum energy dose occurs at a specific depth, allowing delivery of radiation doses that conform to the depth and shape of the target site while minimizing exposure to surrounding normal tissue [26]. This makes PBT notable as a local modality that can effectively treat HCC while minimizing deterioration of surrounding liver function.

In the present study, there were no significant acute toxicities related with PBT. A grade III duodenal ulcer developed in one patient; overall 5.0\% of other gastroduodenal toxicities were mild and acceptable compared to other studies reporting $9.8 \%$ in recent publications using simultaneous integrated boost-PBT for advanced HCC [27]. However, duodenal ulcers were identified at radiation dose of 27.6 CGE in 10 fractions, which is generally considered an acceptable dose. Thus, simple conversion of the previously known limitation dose of photons might be dangerous $[8,28,29]$, and further study of this issue should be conducted.

Almost 70\% infield CR with well-maintained liver function was observed. Non-classic RILD developed in four (4.0\%) patients, which is similar to a recent phase II study using PBT for primary liver cancer [30]. Although higher local control rates of 80\%-90\% for HCC treated with PBT have been reported, data on HCC tumor response after PBT are limited. Kim et al. [31] reported CR rates of $62.5 \%, 57.1 \%$, and $100 \%$ for escalated PBT doses (EQD2) of 65, 71.5, and 78 CGE, respectively, in inoperable HCC using $\mathrm{mRECIST}$. The $\mathrm{CR}$ rate was $69.2 \%$ in our study, which used similar or higher doses 
of irradiation in most cases-EQD2, 75-110 CGE in 72 of 101 patients (71.3\%). However, the evaluation was limited because of the relatively short median follow-up period (4.9 months) and mixed usage of follow-up imaging, CT or MRI according to the physician's preference. A longer follow-up period is needed to evaluate the $C R$ rate and long-term local control more accurately. Kim et al. [31] emphasized that short-term tumor response does not perfectly coincide with long-term local tumor control. A similar phenomenon was also repeated in the study of SABR for HCC [32-34]. In one study, CR rate was $57 \%$ at 3 months, but it continuously increased up to $91.4 \%$ at 1-year [32].

Our study is limited in that it is a single institutional analysis associated with inevitable selection bias. The follow-up period was very short to assess the long-term toxicity or efficacy of PBT. In addition, it is difficult to generalize the results because heterogeneous patients who underwent liver directed PBT with HCC were analyzed. However, it provides useful information about tumor response rate and acute toxicity of PBT, a promising treatment option for HCC management.

In conclusion, PBT showed a favorable CR rate of $69.2 \%$ with acceptable acute toxicity at a median follow-up interval of 4.9 months in $101 \mathrm{HCC}$ patients who were not candidates for or were refractory to standard local modalities. Additional follow-up study of these patients will be conducted. PBT appears to be an effective and safe local modality for such HCC patients.

\section{Conflict of Interest}

No potential conflict of interest relevant to this article was reported.

\section{Acknowledgments}

This research was supported by a Samsung Medical Center grant (No. GF01130081), a Basic Science Research Program through the National Research Foundation of Korea (NRF) funded by the Ministry of Education (No. NRF2015R1D1A1A01060945), and a grant from the Marine Biotechnology Program (No. 20150220) funded by the Ministry of Oceans and Fisheries, Korea.

\section{References}

1. Global Burden of Disease Cancer Collaboration, Fitzmaurice $C$,

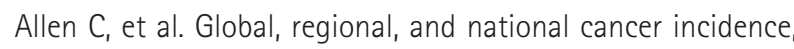

mortality, years of life lost, years lived with disability, and disability-adjusted life-years for 32 cancer groups, 1990 to 2015: a systematic analysis for the global burden of disease study. JAMA Oncol 2017;3:524-48.

2. Kim BH, Lim YS, Kim EY, et al. Temporal improvement in survival of patients with hepatocellular carcinoma in a hepatitis B virus-endemic population. J Gastroenterol Hepatol 2018;33:475-83.

3. Kudo M, Izumi $N$, Ichida $T$, et al. Report of the 19th followup survey of primary liver cancer in Japan. Hepatol Res 2016;46:372-90.

4. Lencioni R, Petruzzi P, Crocetti L. Chemoembolization of hepatocellular carcinoma. Semin Intervent Radiol 2013;30:311.

5. Llovet JM, Real MI, Montana X, et al. Arterial embolisation or chemoembolisation versus symptomatic treatment in patients with unresectable hepatocellular carcinoma: a randomised controlled trial. Lancet 2002;359:1734-9.

6. Cha H, Park HC, Yu Jl, et al. Clinical practice patterns of radiotherapy in patients with hepatocellular carcinoma: a Korean Radiation Oncology Group study (KROG 14-07). Cancer Res Treat 2017;49:61-9.

7. Kim TH, Kim DY, Park JW, et al. Dose-volumetric parameters predicting radiation-induced hepatic toxicity in unresectable hepatocellular carcinoma patients treated with threedimensional conformal radiotherapy. Int J Radiat Oncol Biol Phys 2007;67:225-31.

8. Yu JI, Cho JY, Park HC, Lim DH, Gwak GY, Paik SW. Child-Pugh score maintenance in cirrhotic hepatocellular carcinoma patients after radiotherapy: aspects of gastroduodenal complications. Tumori 2014;100:645-51.

9. Yu Jl, Park HC, Lim DH, Park WY. Predictive factors for ChildPugh score elevation in hepatocellular carcinoma patients treated with conformal radiation therapy: dose-volume histogram analysis. Tumori 2013;99:164-71.

10. Pan CC, Kavanagh BD, Dawson LA, et al. Radiationassociated liver injury. Int J Radiat Oncol Biol Phys 2010;76(3 Suppl):S94-100.

11. Sakurai $H$, Ishikawa $H$, Okumura T. Proton beam therapy in Japan: current and future status. Jpn J Clin Oncol 2016;46:885-92.

12. Bowen SR, Saini J, Chapman TR, et al. Differential hepatic avoidance radiation therapy: Proof of concept in hepatocellular carcinoma patients. Radiother Oncol 2015;115:203-10.

13. Chung K, Han Y, Kim J, et al. The first private-hospital based proton therapy center in Korea; status of the Proton 
Therapy Center at Samsung Medical Center. Radiat Oncol J 2015;33:337-43.

14. Bruix J, Sherman M. Management of hepatocellular carcinoma: an update. Hepatology 2011;53:1020-2.

15. Takamatsu S, Yamamoto K, Maeda Y, et al. Evaluation of focal liver reaction after proton beam therapy for hepatocellular carcinoma examined using Gd-EOB-DTPA enhanced hepatic magnetic resonance imaging. PLoS One 2016;11:e0167155.

16. Jung SH, Yu JI, Park HC, Lim DH, Han Y. A feasibility study evaluating the relationship between dose and focal liver reaction in stereotactic ablative radiotherapy for liver cancer based on intensity change of Gd-EOB-DTPA-enhanced magnetic resonance images. Radiat Oncol J 2016;34:64-75.

17. Lencioni R, Llovet JM. Modified RECIST (mRECIST) assessment for hepatocellular carcinoma. Semin Liver Dis 2010;30:52-60.

18. Korean Liver Cancer Study Group (KLCSG); National Cancer Center, Korea (NCC). 2014 Korean Liver Cancer Study GroupNational Cancer Center Korea practice guideline for the management of hepatocellular carcinoma. Korean J Radiol 2015;16:465-522.

19. Cheng S, Chen M, Cai J; National Research Cooperative Group for Diagnosis and Treatment of Hepatocellular Carcinoma with Tumor Thrombus. Chinese expert consensus on multidisciplinary diagnosis and treatment of hepatocellular carcinoma with portal vein tumor thrombus: 2016 edition. Oncotarget 2017;8:8867-76.

20. Park HC, Yu Jl, Cheng JC, et al. Consensus for radiotherapy in hepatocellular carcinoma from the 5th Asia-Pacific Primary Liver Cancer Expert Meeting (APPLE 2014): current practice and future clinical trials. Liver Cancer 2016;5:162-74.

21. Yu JI, Park JW, Park HC, et al. Clinical impact of combined transarterial chemoembolization and radiotherapy for advanced hepatocellular carcinoma with portal vein tumor thrombosis: an external validation study. Radiother Oncol 2016;118:408-15.

22. Cho JY, Paik YH, Park HC, et al. The feasibility of combined transcatheter arterial chemoembolization and radiotherapy for advanced hepatocellular carcinoma. Liver Int 2014;34:795801.

23. Kokudo T, Hasegawa $K$, Matsuyama $Y$, et al. Survival benefit of liver resection for hepatocellular carcinoma associated with portal vein invasion. J Hepatol 2016;65:938-43.

24. Lee JM, Jang BK, Lee $Y J$, et al. Survival outcomes of hepatic resection compared with transarterial chemoembolization or sorafenib for hepatocellular carcinoma with portal vein tumor thrombosis. Clin Mol Hepatol 2016;22:160-7.

25. Keane FK, Wo JY, Zhu AX, Hong TS. Liver-directed radiotherapy for hepatocellular carcinoma. Liver Cancer 2016;5:198-209.

26. Wilson RR. Radiological use of fast protons. Radiology 1946;47:487-91.

27. Kim DY, Park JW, Kim TH, et al. Risk-adapted simultaneous integrated boost-proton beam therapy (SIB-PBT) for advanced hepatocellular carcinoma with tumour vascular thrombosis. Radiother Oncol 2017;122:122-9.

28. Yoon H, Oh D, Park HC, et al. Predictive factors for gastroduodenal toxicity based on endoscopy following radiotherapy in patients with hepatocellular carcinoma. Strahlenther Onkol 2013;189:541-6.

29. Kim H, Lim DH, Paik SW, et al. Predictive factors of gastroduodenal toxicity in cirrhotic patients after threedimensional conformal radiotherapy for hepatocellular carcinoma. Radiother Oncol 2009;93:302-6.

30. Hong TS, Wo JY, Yeap BY, et al. Multi-institutional phase II study of high-dose hypofractionated proton beam therapy in patients with localized, unresectable hepatocellular carcinoma and intrahepatic cholangiocarcinoma. J Clin Oncol 2016;34:460-8.

31. Kim TH, Park JW, Kim YJ, et al. Phase I dose-escalation study of proton beam therapy for inoperable hepatocellular carcinoma. Cancer Res Treat 2015;47:34-45.

32. Oldrini G, Huertas A, Renard-Oldrini S, et al. Tumor response assessment by $\mathrm{MRI}$ following stereotactic body radiation therapy for hepatocellular carcinoma. PLoS One 2017;12:e0176118.

33. Weiner AA, Olsen J, Ma D, et al. Stereotactic body radiotherapy for primary hepatic malignancies: report of a phase I/II institutional study. Radiother Oncol 2016;121:79-85.

34. Kim JW, Seong J, Lee IJ, Woo JY, Han KH. Phase I dose escalation study of helical intensity-modulated radiotherapybased stereotactic body radiotherapy for hepatocellular carcinoma. Oncotarget 2016;7:40756-66. 